\title{
Effect of Chronic Ethanol Feeding on Glutathione and Functional Integrity of Mitochondria in Periportal and Perivenous Rat Hepatocytes
}

\author{
Carmen García-Ruiz, ${ }^{\star 5}$ Albert Morales, ${ }^{\star}$ Antonio Ballesta, ${ }^{\ddagger}$ Joan Rodés, ${ }^{\star}$ \\ Neil Kaplowitz, and José Carlos Fernández-Checa*łs \\ *Liver Unit, ' Servicio de Bioquímica, Hospital Clinic i Provincial, Departamento de Medicina, Universidad de Barcelona, \\ 08036-Barcelona, Spain; and ${ }^{8}$ Division of Gastrointestinal and Liver Diseases, Department of Medicine, University of Southern \\ California School of Medicine, Los Angeles, California 90033
}

\begin{abstract}
Chronic ethanol feeding selectively impairs the translocation of cytosol GSH into the mitochondrial matrix. Since ethanol-induced liver cell injury is preferentially localized in the centrilobular area, we examined the hepatic acinar distribution of mitochondrial GSH transport in ethanol-fed rats. Enriched periportal (PP) and perivenous (PV) hepatocytes from pair- and ethanol-fed rats were prepared as well as mitochondria from these cells. The mitochondrial pool size of GSH was decreased in both PP and PV cells from ethanol-fed rats either as expressed per $10^{6}$ cells or per microliter of mitochondrial matrix volume. The rate of reaccumulation of mitochondrial GSH and the linear relationship of mitochondrial to cytosol GSH from ethanol-fed mitochondria were lower for both PP and PV cells, effects observed more prominently in the PV cells. Mitochondrial functional integrity was lower in both PP and PV ethanol-fed rats, which was associated with decreased cellular ATP levels and mitochondrial membrane potential, effects which were greater in the PV cells. Mitochondrial GSH depletion by ethanol feeding preceded the onset of functional changes in mitochondria, suggesting that mitochondrial GSH is critical in maintaining a functionally competent organelle and that the greater depletion of mitochondrial GSH by ethanol feeding in PV cells could contribute to the pathogenesis of alcoholic liver disease. (J. Clin. Invest. 1994. 94:193-201.) Key words: ethanol feeding $\cdot$ glutathione $\cdot$ zonal mitochondrial integrity - mitochondrial transport • periportal and perivenous hepatocytes
\end{abstract}

\section{Introduction}

GSH, the most abundant nonprotein thiol in cells, participates in multiple cell functions. As a substrate of GST and GSHperoxidase, GSH constitutes one of the most important lines of cellular defense in the protection and metabolism of xenobiotics and reactive oxygen species (1-5). The bulk of GSH (80-90\%) is found in cytosol, where it is exclusively synthesized in twostep reactions, and in mitochondria $(10-15 \%)$, which is derived

Address correspondence to Dr. Fernández-Checa, Liver Unit, Hospital Clinic i Provincial, Villarroel, 170, 08036-Barcelona, Spain.

Received for publication 19 October 1993 and in revised form 28 February 1994.

J. Clin. Invest.

(C) The American Society for Clinical Investigation, Inc.

0021-9738/94/07/0193/09 \$2.00

Volume 94 , July 1994, 193-201 from the existence of a mitochondrial transporter that translocates GSH from cytosol into the mitochondrial matrix (6-8). The exact mechanism of this transport system is presently unknown, although recent studies from different groups have suggested that either GSH diffuses into mitochondria through a channel (9), is transported by a primary active, high affinity transport system (7), or is the subject of a rapid exchange between cytosol and mitochondria with a $t_{1 / 2}$ of $18 \mathrm{~min}$ (8). The role of GSH in the mitochondria is believed to be critical in the maintenance of vital mitochondrial and cellular functions through the metabolism of reactive oxygen derivatives generated within the mitochondria in the electron transport chain and through the regulation of mitochondrial inner membrane permeability by maintaining $\mathrm{Ca}^{2+}$ homeostasis (10-15). In addition, since mitochondria do not contain catalase, the only exception being heart mitochondria, the GSH redox system is the only defense to metabolize reactive oxygen metabolites $\left(\mathrm{H}_{2} \mathrm{O}_{2}\right)$ produced endogenously in aerobic cells $(16,17)$. Thus, it has been shown in several cell types and organs that depletion of mitochondrial GSH results in cell injury and death, which is prevented by the selective increase of the mitochondrial pool of $\operatorname{GSH}(8,10,11)$.

Previous studies using the Lieber-DeCarli model in wellnourished rats have shown that chronic ethanol feeding substantially decreases the mitochondrial pool of GSH either in isolated hepatocytes or in vivo as a result of an impaired transport activity of GSH from cytosol into the mitochondrial matrix $(8,18$, 19). The lower mitochondrial GSH found in alcoholic cells increases their susceptibility to the lethal effects of oxidants, which is corrected by increasing the content of GSH using GSHmonoethyl ester (8). Recently, using an experimental model of alcohol-induced fibrosis and necrosis by intragastric alcohol infusion, we have shown that ethanol selectively and progressively decreases the mitochondrial pool of GSH, preceding the onset and progression of liver disease associated with mitochondrial lipid peroxidation and increased serum alanine aminotransferase levels (20).

There is a great deal of evidence pointing to the toxic effects of ethanol on mitochondria, manifested as functional and ultrastructural changes, observed in ethanol-fed rats and alcoholic patients. However, there are no data regarding the zonal effects of ethanol on mitochondria functionality along the liver acinus (21-25). Since ethanol causes preferential necrosis and fibrosis in the centrilobular zone of the liver (26), the purpose of this study was to examine the hepatic acinar distribution of mitochondrial GSH in control and ethanol-fed rats and the relationship of changes to mitochondria functionality. The goals are to better define both the effects of ethanol on the hepatic GSH defense and the significance of such changes in contributing to liver dysfunction and alcoholic liver disease. 


\section{Methods}

Materials and animals. ADP, antimycin A, GSH, oxidized glutathione, collagenase type IV, digitonin, mannitol, oligomycin, rotenone, $N, N, N^{\prime}, N^{\prime}$-tetramethyl-p-phenylenediamine (TMPD), ${ }^{1}$ and carbonyl cyanide-m-chlorophenyl-hydrazone (m-CCCP) were purchased from Sigma Chemical Co. (St. Louis, MO). ${ }^{14} \mathrm{C}$-Carboxylic acid inulin $(9.4$ $\mathrm{mCi} / \mathrm{mmol}),{ }^{3} \mathrm{H}_{2} \mathrm{O}$, and tetra $\left({ }^{3} \mathrm{H}\right)$ phenylphosnonium bromide $(33 \mathrm{Ci} /$ mmol) were purchased from Amersham Corp. (Arlington Heights, IL). Other reagents were of analytical grade.

Male Sprague-Dawley rats weighing 150-170 g were pair fed the original Lieber-DeCarli liquid diet (27) (PanLab, Barcelona, Spain) as described previously $(18,19)$. In the ethanol-fed liquid diet, the caloric contribution of protein, lipids, carbohydrates, and ethanol was 18,35 , 11 , and $36 \%$ of calories, respectively. The pair-fed group received an isocaloric mixture with maltose dextrin substituted for ethanol $(18,19)$. For a more detailed description of the composition of the liquid diet see reference 27. Animals were pair fed for up to 4 wk unless otherwise stated.

Isolation of periportal $(P P)$ and perivenous $(P V)$ hepatocytes. Hepatocytes selectively enriched in either population of cells were obtained based on the digitonin/collagenase perfusion technique with certain modifications as detailed below (28). The livers of anesthesized pairand ethanol-fed rats were initially perfused in situ with Hanks' buffer at a flow rate of $20 \mathrm{ml} / \mathrm{min}$. For zone-specific cell destruction, digitonin ( $2.5 \mathrm{mM}$ prepared in boiled Hanks' buffer) was infused for $10-15$ or $30-40 \mathrm{~s}$ in the prograde or retrograde direction to prepare PV and PP hepatocytes, respectively. Immediately after the infusion of digitonin, Hanks' buffer was infused through the opposite cannula for 2-3 min. This was followed by a collagenase perfusion with Hanks' buffer supplemented with $2.5 \mathrm{mM} \mathrm{CaCl}$ and collagenase $(30-35 \mathrm{mg} / 150 \mathrm{ml})$ according to Moldeus (29) and handled as described previously $(8,18$, 19). Initial viability ascertained by trypan blue exclusion $(0.2 \%)$ was $95 \pm 3 \%$ and was similar for PP or PV cells from pair- or ethanol-fed rats. Enrichment of cell populations was determined by enzyme activities of zone-specific markers such as succinic and lactate dehydrogenases (SDH and LDH, respectively) for the periportal region and glutamine synthetase (GS) for the perivenous region $(28,30,31)$.

Incubation and fractionation of $P P$ and $P V$ cells. Cells were manipulated to achieve a range of cellular GSH concentrations as described previously $(8,18,19)$. To decrease cell GSH levels below the physiological range, cells were incubated with moderate doses of DEM (25-100 $\mu \mathrm{M}$ ) in Krebs-Henseleit buffer for $15 \mathrm{~min}$ followed by extensive (three to four times) washings with buffer. Additionally, to increase cell GSH levels above physiological values and to examine the time-dependent accumulation of newly synthesized GSH and transport of cytosol GSH into mitochondria, cells were incubated in modified Fisher's medium with $1 \mathrm{mM}$ methionine as GSH precursor for up to $3 \mathrm{~h}$ under $95 \% \mathrm{O}_{2}$ and $5 \% \mathrm{CO}_{2}$. At every time point, an aliquot of cells was taken, and cells were fractionated into cytosol and particulate fraction by selectively permeabilizing the plasma membrane with digitonin and subsequent centrifugation through oil layer as described previously $(8,18,19)$. Parallel aliquots were taken for fractionation of cells, except that the bottom of the oil layer contained $40 \%$ glycerol instead of $10 \%$ TCA to examine the enzymatic content of SDH and LDH to follow the fractionation and cross-contamination as described previously $(8,18)$. Aliquots of the soluble and particulate fraction were kept for determination of GSH, ATP, and enzyme markers as described below.

Mitochondrial isolation and functional integrity. Mitochondria from

1. Abbreviations used in this paper: ACR, acceptor control ratio; GS, glutamine synthetase; LDH, lactate dehydrogenase; m-CCCP, carbonyl cyanide-m-chlorophenyl-hydrazone; $\mathrm{PP}$, periportal; $\mathrm{PV}$, perivenous; SDH, succinic dehydrogenase; TMPD, $N, N, N^{\prime}, N^{\prime}$-tetramethyl-p-phenylenediamine; TPP, tetraphenylphosphonium bromide; UCR, uncoupler control ratio.
PP and PV zones were isolated by centrifugation through a discontinuous Percoll gradient $(19,33,41$, and $52 \%)$ as described previously (8, 32). Plasma membrane of intact PP and PV cells isolated as described above was lysed by either homogenization at $15-10 \%$ (assuming that $10^{8}$ cells are equivalent to $1 \mathrm{~g}$ of liver) in mannitol buffer $(210 \mathrm{mM}$ mannitol, $60 \mathrm{mM}$ sucrose, $10 \mathrm{mM} \mathrm{KCl}, 10 \mathrm{mM}$ sodium succinate, 1 $\mathrm{mM}$ ADP, $0.25 \mathrm{mM}$ DTT, $0.1 \mathrm{mM}$ EGTA in $10 \mathrm{mM}$ Hepes, pH 7.4) $8-10$ times (up/down) with the pestle driven at $800 \mathrm{rpm}$ or by exposure of cells to $0.0075 \%$ digitonin solution for $1 \mathrm{~min}$ followed by three to four washes with Percoll medium ( $400 \mathrm{~g}$ for $20 \mathrm{~s}$ each time) at room temperature. Homogenate was layered in Percoll gradient and centrifuged for $1 \mathrm{~min}$ at 19,000 rpm in an A12.24 rotor (Kontron Instruments, Milan, Italy). The mitochondrial band was collected with a Pasteur pipette, centrifuged in mannitol medium for $15 \mathrm{~min}$ at $8,000 \mathrm{~g}$, resuspended at $10-20 \mathrm{mg} / \mathrm{ml}$ protein, and kept in ice for oxygen consumption determination.

The rate of oxygen consumption was measured polarographically with an Oxygraph (Gilson Electronics, Midleton, WI) equipped with a Clark-type electrode attached to a thermoregulated reaction vessel with a magnetic stirrer. An aliquot of mitochondrial suspension $(0.1 \mathrm{ml}, 1-$ $2 \mathrm{mg}$ protein) was added to $1.2 \mathrm{ml}$ of respiration medium $(225 \mathrm{mM}$ sucrose, $5 \mathrm{mM} \mathrm{MgCl}_{2}, 10 \mathrm{mM} \mathrm{KH}_{2} \mathrm{PO}_{4}, 20 \mathrm{mM} \mathrm{KCl}, 10 \mathrm{mM}$ Tris, and $5 \mathrm{mM}$ Hepes, $\mathrm{pH}$ 7.4). Glutamate and malate (final concentration $5 \mathrm{mM}$ each) or succinate (final concentration $17 \mathrm{mM}$ ) were added as substrates for complex I and complex II, respectively. State 3 respiration was determined by addition of ADP to the mixture $(0.7 \mathrm{mM})$. Small aliquots $(10 \mu \mathrm{l})$ of stock ethanolic solutions of oligomycin and $\mathrm{m}-\mathrm{CCCP}$, at final concentrations of $13 \mu \mathrm{g} / \mathrm{ml}$ and $13 \mu \mathrm{M}$, respectively, were added to the reaction vessel. The rate of oxygen consumption of energized mitochondria in the presence of ADP over the absence of ADP is defined as the acceptor control ratio (ACR) (state 3/state 4 respiration). Maximal electron transport rate was determined in the presence of the uncoupler, $\mathrm{m}-\mathrm{CCCP}$. The ratio of oxygen consumed in the presence of uncoupler/ oligomycin is defined as the uncoupler control ratio (UCR). Both ACR and UCR are indicators of mitochondrial integrity: the higher the value, the greater the coupling of oxidative phosphorylation. In some instances, to determine oxygen consumption by selective electron donation to cytochrome $c$, TMPD $(0.2 \mathrm{mM}$, plus ascorbate $1 \mathrm{mM})$ was used as an artificial electron donor after inhibition of electron flow from complex III to cytochrome $c$ with antimycin A $(5 \mu \mathrm{M})$.

Maximal synthetic capacity of GSH. The maximal capacity of cells to synthesize GSH was determined in a cell-free system using a GSHspecific fluorescent probe, monochlorobimane, using cysteine as the GSH precursor as described in detail previously (33). Cytosol from PV or PP cells was dialyzed overnight at $4^{\circ} \mathrm{C}$ to deplete GSH to minimize the feedback inhibitory effect of GSH on the $\gamma$-glutamylcysteine synthetase. The synthetic rate of GSH was determined as the net rate of fluorescence increase over time after subtracting the BSO-inhibitable fluorescence signal (33).

Determination of mitochondrial matrix volume and membrane potential. Mitochondria from PP or PV region of rat liver were incubated at $3 \mathrm{mg} / \mathrm{ml}$ in a medium consisting of $120 \mathrm{mM} \mathrm{KCl}, 5 \mathrm{mM}$ Hepes$\mathrm{KOH}, \mathrm{pH} 7.0,5 \mathrm{mM}$ succinate (potassium salt), $1 \mathrm{mM}$ EGTA, $5 \mu \mathrm{M}$ rotenone, nigericin ( $100 \mathrm{pmol} / \mathrm{mg}$ mitochondrial protein), $1 \mu \mathrm{M}$ bromide salt (TPP), and ${ }^{3} \mathrm{H}_{2} \mathrm{O}(1.0 \mu \mathrm{Ci} / \mathrm{ml})$ to determine matrix volume after subtraction of trapping estimated by addition of $\left[\mathrm{U}-{ }^{14} \mathrm{C}\right]$ inulin $(0.1 \mu \mathrm{Ci} /$ $\mathrm{ml})$ or $\left[{ }^{3} \mathrm{H}\right] \mathrm{TPP}(0.2 \mu \mathrm{Ci} / \mathrm{ml})$ for membrane potential determination, at $25^{\circ} \mathrm{C}(34) .1-\mathrm{ml}$ aliquots were taken at timed intervals and centrifuged in a microfuge (Beckman Instruments, Inc., Fullerton, CA) for 1 min at room temperature to pellet the mitochondria. $500 \mu \mathrm{l}$ of supernatant was removed, added to $4.0 \mathrm{ml}$ of scintillant, and counted. Residual supernatant was removed with a tissue. The mitochondrial pellet was resuspended in $40 \mu \mathrm{l}$ Triton X-100 (20\% vol/vol) and counted. Mitochondrial membrane potential differences were calculated from the ratio of concentration of TPP in mitochondria and in the external medium, according to the Nernst equation:

$\mathrm{Em}=-\mathrm{RT} / \mathrm{F} \ln \left[\mathrm{TPP}^{+}\right] \mathrm{in} /\left[\mathrm{TPP}^{+}\right] \mathrm{ex}$. 
Table I. Marker Enzyme Activities and Cell Volume of PP and PV Hepatocytes Isolated from Control and Ethanol-fed Rats

\begin{tabular}{|c|c|c|c|c|c|c|}
\hline & \multicolumn{2}{|c|}{ PP } & \multicolumn{2}{|c|}{ PV } & \multicolumn{2}{|c|}{ PP/PV } \\
\hline & Control & ETOH & Control & ETOH & Control & ЕTOH \\
\hline & \multicolumn{6}{|c|}{ umol/min per $10^{8}$ cells } \\
\hline \multicolumn{7}{|l|}{ Markers } \\
\hline LDH & $351 \pm 47.5$ & $372 \pm 32.1$ & $226 \pm 24.1$ & $267 \pm 43.1$ & $1.55 \pm 0.3$ & $1.39 \pm 0.4$ \\
\hline SDH & $0.37 \pm 0.45$ & $0.41 \pm 0.52$ & $0.22 \pm 0.39$ & $0.27 \pm 0.43$ & $1.65 \pm 0.09$ & $1.51 \pm 0.08$ \\
\hline Glutamine synthase & $0.08 \pm 0.9$ & $0.05 \pm 1.3$ & $4.57 \pm 0.39$ & $5.15 \pm 0.45$ & $0.071 \pm 0.002$ & $0.009 \pm 0.001$ \\
\hline Cell water $\left(\mu \nu 10^{6}\right.$ cells $)$ & $3.6 \pm 0.4$ & $4.4 \pm 0.3 *$ & $4.1 \pm 0.3$ & $5.8 \pm 0.4^{\ddagger \S}$ & $0.87 \pm 0.08$ & $0.75 \pm 0.09$ \\
\hline
\end{tabular}

Results are the mean \pm SD of $n=4$ cell preparations from pair- and ethanol-fed rats. ${ }^{\ddagger} P<0.05$ vs PP cells from ethanol-fed rats; ${ }^{8} P<0.05$ vs PV cells from pair-fed rats; and $* P<0.05$ vs PP cells from pair-fed rats. Comparisons were done by ANOVA.

Assays. GSH was determined by either the recycling method (35) or by HPLC as described previously in detail $(8,36)$. ATP levels were determined by HPLC using a reverse-phase column according to the method of Jones (37). LDH was determined by a commercial kit (Sigma Chemical Co.), and SDH was determined according to the method of Hatefi (38). GS was measured as described (39). Cell volume and diameter of pair- and ethanol-fed PP and PV hepatocytes were determined as the ${ }^{3} \mathrm{H}_{2} \mathrm{O}$ volume after subtraction of trapping $\left(\left[{ }^{14} \mathrm{C}\right]\right.$ inulin) and by particle counting (Channelyzer; Coulter Corp., Hialeah, FL), respectively, as described previously $(8,40)$. Alcohol dehydrogenase was determined

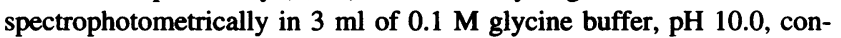
taining $2.4 \mathrm{mM} \mathrm{NAD}^{+}$and $33 \mathrm{mM}$ ethanol. Low $K_{\mathrm{m}}$ acetaldehyde dehydrogenase was assayed in $3 \mathrm{ml}$ of $0.1 \mathrm{M}$ sodium pyrophosphate, $\mathrm{pH}$ 9.5, containing $2.4 \mathrm{mM} \mathrm{NAD}^{+}, 25 \mu \mathrm{M}$ acetaldehyde, and $0.15 \mathrm{mM} \mathrm{4-}$ methylpyrazole. Statistical analyses for multiple comparisons of the mean between groups and between cell populations were made by oneway ANOVA followed by Fisher's test.

\section{Results}

Separation and features of $P P$ and $P V$ cells from pair- and ethanol-fed rats. Selective destruction of zonal hepatocytes by digitonin, determined by the direction of infusion, detergent concentration, and time of exposure, has been used to prepare highly enriched PP and PV hepatocytes after lysing PV and PP cell populations, respectively. To monitor the degree of selective destruction and lack of cross-contamination of the zonally enriched hepatocytes, we have determined the activities of enzymatic markers of either zone. As shown in Table I, the activities of LDH and SDH, enzymes predominantly present in the periportal area $(26,29,30)$, were higher in PP hepatocytes than in PV cells, so that the PP/PV ratio was $>1$. However, the activity of GS, which is found exclusively in cells next to the hepatic vein, was higher in PV than in PP cells, with a PP/PV ratio $<0.02$. Furthermore, when the same protocol was applied to ethanol-fed rats, similar marker activities for zonal hepatocytes were found compared with pair-fed rats, indicating qualitatively similar enrichment of PP and PV cells from pair- and ethanolfed rats. The initial viability of either type of cell was at least 90\% as estimated by trypan blue exclusion and did not differ between pair- and ethanol-fed rats (not shown). Since ethanol feeding results in enlargement of cells due to retention of water and proteins (19), we determined if there was a zonal preference in the ethanol-induced enlargement of PP and PV cells. In pairfed cells, PV hepatocytes contained slightly greater cell water content than PP cells (by 15\%). Ethanol feeding resulted in a significant enlargement of both PP and PV cells (Table I). Determination of cell diameter by particle counting (Channelyzer; Coulter Corp.) resulted in qualitatively similar zonal cellular increases by ethanol feeding compared with pair-fed cells (data not shown).

Table II shows the aldehyde dehydrogenase activity in PP and PV cells from pair- and ethanol-fed rats. The PV cells displayed a lower activity compared with PP cells from pairfed rats. In ethanol-fed cases, activity was lowered in both PP and PV cells.

Basal cytosol and mitochondrial GSH status and transport of GSH from cytosol into mitochondria in PP and PV cells. To determine the GSH content in cytosol and mitochondrial fractions, PP and PV cells were fractionated by exposure to digitonin followed by centrifugation through Percoll gradient as described previously $(18,19)$. Determination of LDH and SDH activities in both compartments indicated an optimal fractionation of PP and PV cells into cytosol and mitochondria, with similar results for PP and PV cells from ethanol-fed rats (not shown). As shown in Fig. 1, cytosol GSH content in PP and PV pair-fed cells expressed per number of cells was not significantly different and was unchanged by chronic ethanol feeding (Fig. $1 A$ ). Since ethanol feeding increases the cell water content in both acinar zones (Table I) and assuming that the cytosol water is increased to the same proportion as the cell water increment, correcting the cytosol GSH content by microliter of cell water resulted in a significant decrease in cytosol GSH concentration in ethanol-fed PV cells (Fig. 1 B). The content of GSH in mitochondria from pair-fed cells was distributed evenly between PV and PP cells (Fig. 1 C). In contrast,

Table II. Acetaldehyde Dehydrogenase Activity of PP and PV Cells from Pair-fed and Ethanol-fed Rats

\begin{tabular}{lllll}
\hline & & & \multicolumn{2}{c}{ PV } \\
\cline { 4 - 5 } Control & ETOH & Control & ETOH \\
\hline & nmolmin per $10^{8}$ cells & \\
$194 \pm 19$ & $144 \pm 15$ & $142 \pm 20^{*}$ & $99 \pm 21^{* \pm}$ \\
\hline
\end{tabular}

Results are the mean \pm SD of $n=4$ cell preparations from pair- and ethanol-fed rats. $\quad * P<0.05$ vs their corresponding PP cells and ${ }^{\ddagger} P$ $<0.05$ vs PV cells from pair-fed rats by ANOVA. 

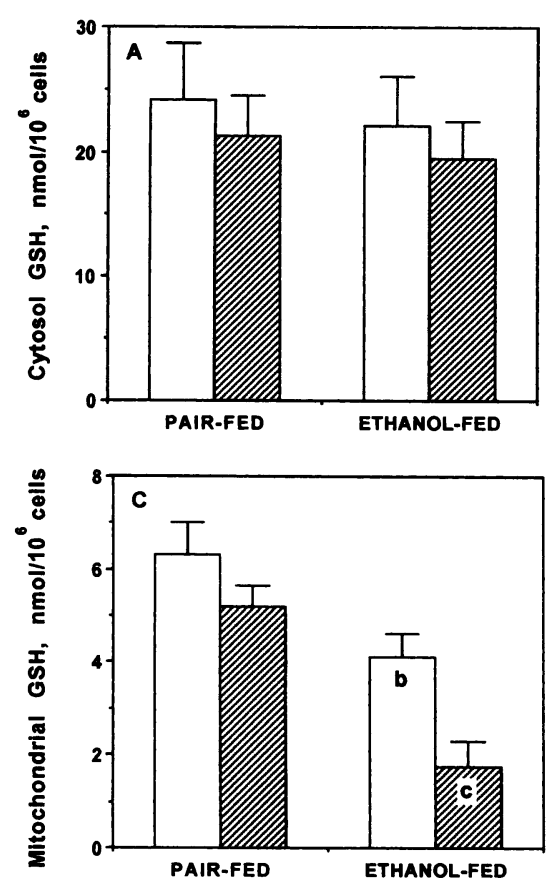
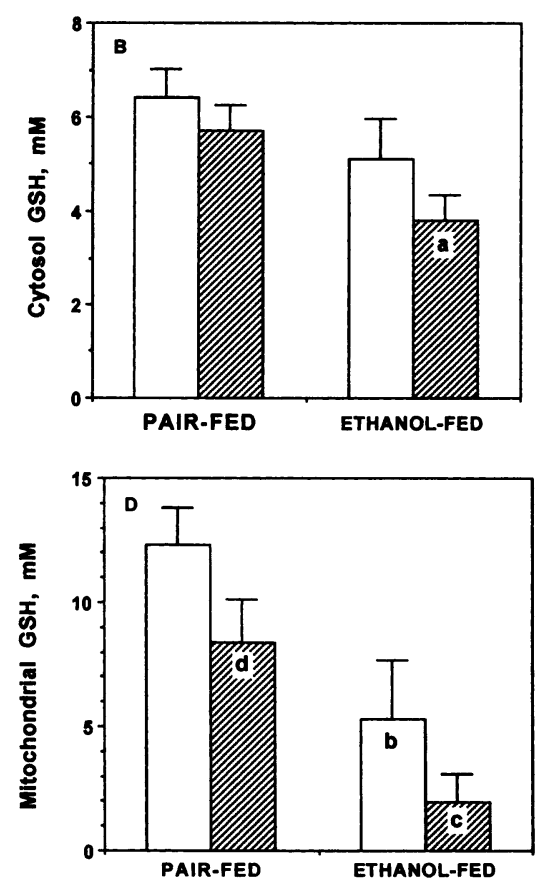

Figure 1. Basal GSH content of cytosol and mitochondrial fractions from PP and PV hepatocytes. PP (solid bars) and PV (hatched bars) hepatocytes from pair-and ethanol-fed rats were isolated as described in Methods. Isolated hepatocytes were then fractionated by exposure to digitonin and subsequent centrifugation through a discontinuous Percoll gradient to isolate a cytosol $(A$ and $B)$ (density, 1.04-1.05 g/ $\mathrm{ml}$ ) and a mitochondrial ( $C$ and $D$ ) (density, $1.85-1.95 \mathrm{~g} / \mathrm{ml}$ ) fraction, and their GSH content was determined by the Tietze method (35). The content of GSH is expressed as per $10^{6}$ cells or as absolute concentration (millimolar) using cell water and mitochondrial matrix volume. Results are the mean \pm SD of $n=4$ preparations (animals) per group. $a, P<0.05 \mathrm{vs} \mathrm{PV}$ pair-fed values; $b, P<0.05$ vs PP pair-fed; $c$, $P<0.05$ vs PP ethanol-fed and $d, P<0.05$ vs PP pair-fed. Comparisons were done by ANOVA. mitochondrial GSH from both zones was depleted significantly by ethanol feeding, an effect that was significantly $(P<0.05$, by ANOVA) greater in PV than in PP ethanol-fed cells $(63 \pm 6$ and $34 \pm 7 \%$ decrease, respectively). To appreciate the zonal distribution of mitochondrial GSH in terms of absolute concentration, we determined the matrix volume of PP and PV mitochondria from pair- and ethanol-fed rats. PV mitochondria from pair-fed rats are moderately larger than those from the PP zone $\left(0.61 \pm 0.05\right.$ and $0.55 \mu \mathrm{l} / 10^{6}$ cells) because they are significantly enlarged by ethanol feeding $\left(0.64 \pm 0.07\right.$ and $0.88 \pm 0.08 \mu \mathrm{l} / 10^{6}$ cells for PP and PV ethanol-fed mitochondria, respectively). The concentration of GSH in mitochondria expressed per microliter of mitochondrial matrix volume is moderately lower in PV zone from pair-fed rats than PP area compared with the expression of results per $10^{6}$ cells. Chronic ethanol feeding decreased the mitochondrial GSH concentration by $55 \pm 7$ and $76 \pm 6 \%$ in PP and PV zones, respectively (Fig. $1 D$ ).

To discern if the lower cytosol GSH concentration in PV ethanol-fed cells was because of an impairment in the synthesis of GSH, we determined the repletion rate of cytosol GSH using methionine $(1 \mathrm{mM})$ as GSH substrate. As shown in Fig. $2 \mathrm{~A}$, the increase over time of cytosol GSH was similar in PP and PV cells from pair- and ethanol-fed rats. To unequivocally determine the inherent capacity of the acinar cells to synthesize GSH and to estimate the effect by ethanol feeding, we examined the synthetic capacity of GSH in a GSH-depleted cell-free system where the cofactors and substrates (cysteine in addition to glycine and glutamate) needed for GSH synthesis were provided without limitation. No difference in the maximal GSH synthetic rate between PP and PV cells from pair- or ethanol-fed cells was observed (Fig. 3). Similar results were obtained when GSH synthesis was monitored using $\gamma$-glutamylcysteine as substrate (not shown). Thus, these results indicate that the $\gamma$-glutamylcysteine synthetase and GSH synthetase operated similarly for either zone area and that ethanol feeding did not impair the GSH synthetic capacity. We assume that the lower GSH concen- tration in PV cells from ethanol-fed rats simply reflects the effect of cell swelling on the steady state balance between precursor concentration, enzyme concentration, and enzyme inhibition by the GSH concentration.

Since mitochondrial GSH is derived from cytosol by a transport system, we determined the rate of accumulation by mitochondria of newly synthesized cytosol GSH. Fig. $2 B$ shows that the accumulation over time of GSH into mitochondria from pair-fed rats was similar for PP and PV zones (3.8 \pm 0.02 and

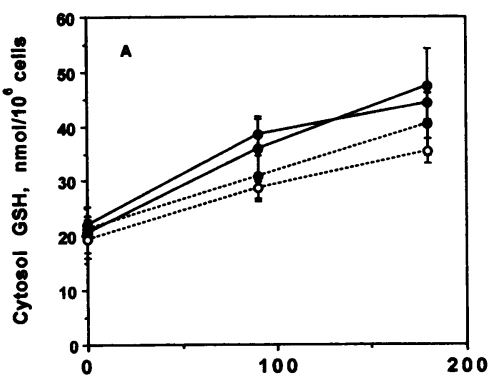

Figure 2. Time-dependent accumulation of GSH in cytosol $(A)$ and mitochondria $(B)$ of PP and PV hepatocytes. Isolated $\mathrm{PP}$ (closed circles) and PV (open circles) hepatocytes from pair-fed (continuous lines) and ethanol-fed (dashed lines) rats were incubated in modified Fisher's medium for up to $3 \mathrm{~h}$ supplemented with 1 $\mathrm{mM}$ methionine. At each time point, a 1-ml aliquot was taken, hepatocytes were fractionated into cytosol $(A)$ and mitochondria $(B)$ by digitonin, and GSH was determined in the TCA extracts. In some cases, samples were derivatized and analyzed by HPLC for confirmation

(36). Results are the mean \pm SD of $n=4$ cell preparations. The rate of accumulation of ethanol-fed cells is significant versus pair-fed values by ANOVA. 


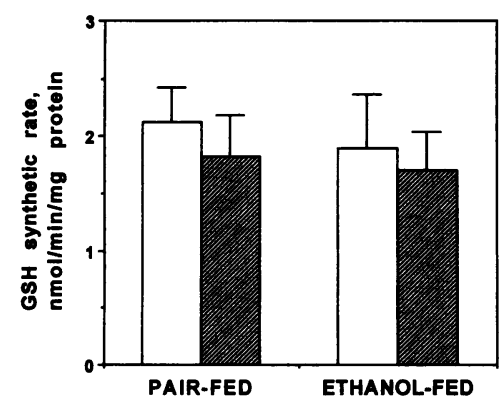
GSH by avoiding its feedback inhibition. GSH synthetic rate was determined in the fluorometer cuvette by adding all the cofactors and precursors needed for GSH synthesis in the presence of $100 \mu \mathrm{M}$ monochlorobimane, as described in detail (33). Results are the mean \pm SD of $n=4$ for pair- and ethanol-fed cytosol preparations.

$3.5 \pm 0.03 \mathrm{nmol} / 3 \mathrm{~h} / 10^{6}$ cells). Ethanol feeding resulted in a lower accumulation of GSH in either the PP or the PV zone $\left(2.8 \pm 0.3\right.$ and $1.2 \pm 0.2 \mathrm{nmol} / 3 \mathrm{~h} / 10^{6}$ cells) compared with pairfed mitochondria, which were significantly lower in PV compared with PP ethanol-fed mitochondria. To exclude a threshold phenomenon and to confirm that this defect in the mitochondrial transport of GSH by ethanol feeding is observed in a wide range of cytosol concentrations, we related the mitochondrial to the cytosol GSH pool for both PP and PV cells from pair- and ethanol-fed rats. As shown in Fig. 4, increasing GSH of pairfed cytosol resulted in a linear increase of mitochondrial GSH
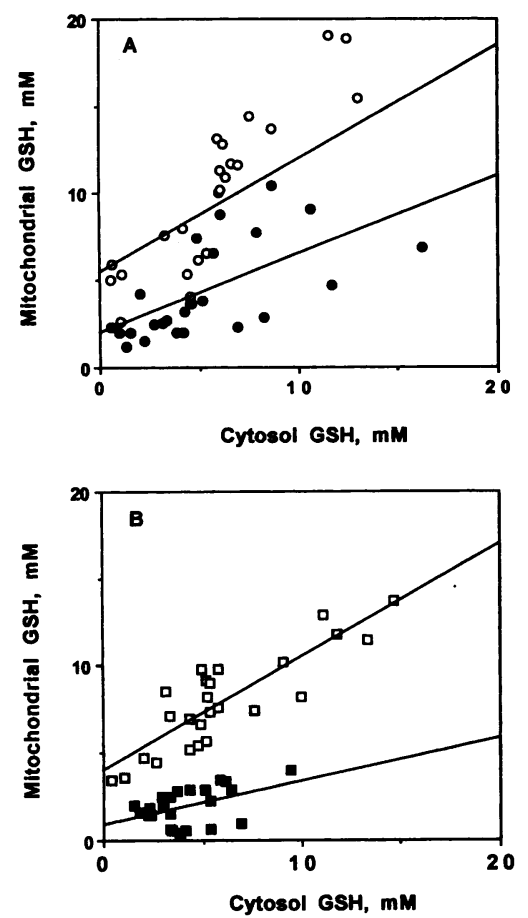

Figure 4. Relationship between cytosol and mitochondrial GSH content from zonal hepatocytes from pair- and ethanolfed rats. PP $(A)$ and PV $(B)$ hepatocytes from pair-(open symbols) and ethanol-fed rats (closed symbols) were isolated by the digitonin/collagenase perfusion technique as described in Methods. To obtain a wide range of cytosol GSH concentration, cells were either untreated, incubated in Fisher's medium in the presence of methionine $(1 \mathrm{mM})$ for 3 $h$, or depleted of GSH by incubation of cells with DEM (25-100 $\mu \mathrm{M})$ for 15 min followed by extensive washing to remove excess DEM. After these maneuvers, cells

were fractionated into cytosol and mitochondria, and GSH was determined in the TCA-soluble portion. The solid lines were determined by linear regression: $\mathrm{y}=5.1+0.12 \mathrm{x}, r=0.731$ and $\mathrm{y}=2.1+0.078 \mathrm{x}$, $r=0.537$ from pair-and ethanol-fed PP cells and $\mathrm{y}=4.7+0.087 \mathrm{x}, r$ $=0.810$ and $\mathrm{y}=1.4+0.029 \mathrm{x}, r=0.611$ from pair-and ethanol-fed PV cells, respectively. Results are from $n=4$ cell preparations for pairand ethanol-fed rats with multiple incubations for each group.
A PP Pair-fed

C PP Etoh-fed

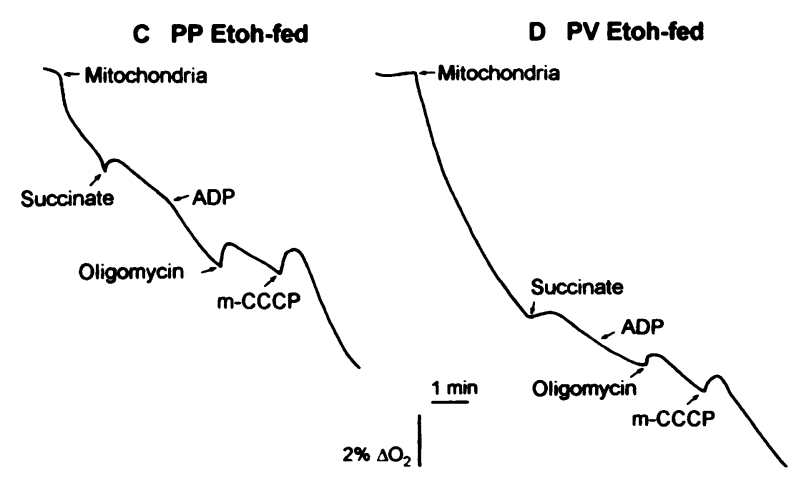

$2 \% \Delta \mathrm{O}_{2} \mid \frac{1}{1}$

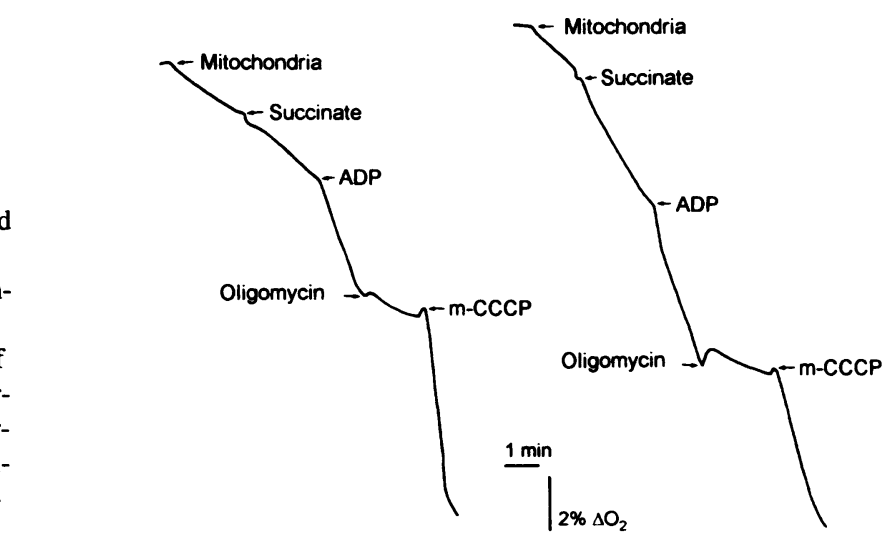

B PV Pair-fed

Figure 5. Representative oxygen electrode tracings showing oxygen uptake rates from PP and PV mitochondria. Mitochondria were prepared from PP and PV hepatocytes from pair-and ethanol-fed (Etoh-fed) rats by a discontinuous Percoll gradient centrifugation after selective rupturing of the plasma membrane by homogenization or incubation with digitonin. Mitochondria were added to the respiration medium on an Oxygraph as described in Methods to record oxygen uptake in the presence of succinate $(17 \mathrm{mM})$, ADP $(0.7 \mathrm{mM})$, oligomycin $(13 \mu \mathrm{g} /$ $\mathrm{ml})$, and $\mathrm{m}$-CCCP $(13 \mu \mathrm{M})$. Qualitatively similar oxygen uptake profiles were obtained when mitochondria were energized with glutamate and malate ( $5 \mathrm{mM}$ each). The ratio of oxygen consumed in the presence of ADP over the absence of ADP is defined as the ACR, and the UCR is the ratio of oxygen consumed in the presence of the uncoupler over that of oligomycin.

for PP and PV cells. In contrast, the slopes of the relationship of mitochondrial to cytosol GSH from ethanol-fed mitochondria were significantly lower for both PP and PV compared with pair-fed cells by 35 and 70\%, respectively. Again, the PV ethanol cells exhibited significantly lower slope versus ethanol PP cells.

Mitochondrial GSH and functional integrity from pair-and ethanol-fed rats. Larger quantities of mitochondria were prepared by centrifugation of lysed PP and PV cells in Percoll density gradient (8). SDH enrichment in the final mitochondrial PP and PV preparation ranged from four to five times relative to the homogenate and was comparable between pair- and ethanol-fed preparations (data not shown). Energized PP and PV mitochondria using succinate as oxidizable substrate were used to determine the oxygen consumption at different states; representative tracings are shown in Fig. 5. Quantitation of the oxygen tracings to determine parameters of mitochondrial functional integrity such as ACR and UCR is shown in Table III. 
Table III. States of Oxygen Consumption of PP and PV Energized Mitochondria from Pair- and Ethanol-fed Rats

\begin{tabular}{|c|c|c|c|c|c|c|}
\hline & State 4 & State 3 & +Oligo & $+\mathrm{m}-\mathrm{CCCP}$ & ACR & UCR \\
\hline & \multicolumn{6}{|c|}{ nmol $O_{2} /$ min per mg protein } \\
\hline \multicolumn{7}{|l|}{ PP } \\
\hline Pair-fed & $20.4 \pm 3.3$ & $126.9 \pm 23.5$ & $15.2 \pm 1.0$ & $141.3 \pm 21.9$ & $6.3 \pm 0.4$ & $9.3 \pm 1.1$ \\
\hline Ethanol-fed & $27.5 \pm 4.9$ & $97.8 \pm 8.8^{*}$ & $22.5 \pm 2.3$ & $110.2 \pm 11.8^{*}$ & $3.7 \pm 1.0^{*}$ & $5.1 \pm 1.6^{*}$ \\
\hline \multicolumn{7}{|l|}{ PV } \\
\hline Pair-fed & $16.8 \pm 4.7$ & $94.3 \pm 13.4$ & $13.7 \pm 2.8$ & $110.1 \pm 25.6$ & $5.8 \pm 0.9$ & $8.3 \pm 1.2$ \\
\hline Ethanol-fed & $48.9 \pm 5.2^{* \ddagger}$ & $57.8 \pm 7.9^{* \ddagger}$ & $34.8 \pm 5.1^{* \pm}$ & $69.8 \pm 9.3^{* \ddagger}$ & $1.2 \pm 0.1^{* \ddagger}$ & $2.0 \pm 0.6^{* \pm}$ \\
\hline
\end{tabular}

Results are the mean \pm SD of $n=4$. Oxygen consumption rates at different states were induced by adding succinate, ADP, oligomycin, and $\mathrm{m}$ CCCP. See Fig. 5 for representative examples. ${ }^{*} P<0.05$ vs pair-fed values, and ${ }^{\ddagger} P<0.05$ vs PP values by ANOVA.

Whereas ACR and UCR values did not differ between PP and PV pair-fed mitochondria, both PP and PV ethanol-fed mitochondria showed significantly lower values of ACR and UCR, with both values significantly more diminished in the PV zone compared with the PP zone. Decreases of the ACR values after ethanol feeding were accounted for by a decrease in state 3 respiration, indicating a lower rate of ATP synthesis for both PP and PV hepatic zones. In addition, in zone PV, there was a significant increase in the resting rate of respiration (state $4, P$ $<0.05$ vs PP values, by ANOVA) after ethanol feeding, suggesting that mitochondria in the PV zone are highly uncoupled to the oxidative phosphorylation. The maximal electron transport rate was decreased significantly in both PP and PV mitochondria after ethanol feeding, indicating the presence of functionally altered sites of electron transport between complex II and cytochrome oxidase. Similar oxygen tracings and UCR values were obtained using glutamate plus malate and TMPD plus ascorbate as electron donors at the level of complex I and III, respectively (not shown). The changes observed in the coupling of oxidative phosphorylation as a result of ethanol feeding are reflected in significantly decreased steady state ATP levels in hepatocytes of ethanol-fed rats compared with pair-fed rats; this was most pronounced in the PV area (Table IV). In addition, there was a decrease in the mitochondrial membrane potential with depolarization more predominantly seen in the PV area (Table IV).

Relationship of mitochondrial GSH depletion and functional integrity with duration of ethanol feeding. As shown on Table $\mathrm{V}$ with increased duration of ethanol feeding from 1 to $4 \mathrm{wk}$, a progressive depletion of mitochondrial GSH and impairment of functional integrity were observed. As early as 1 wk of ethanol feeding, PV mitochondria exhibited a $27 \%$ decrease in GSH pool size without a change in UCR values. Longer exposure to ethanol resulted in a further decline in mitochondrial GSH and the appearance of functional alterations of mitochondria in PV hepatocytes. Changes in mitochondria from PP ethanol-fed cells lagged behind those of PV cells and were significantly less severe. As with PV cells, a significant decrease in mitochondrial GSH preceded the decrease in UCR value.

Fig. 6 shows the correlation between mitochondrial GSH content and its functional integrity expressed as UCR. There was a close correlation between UCR and mitochondrial GSH either when expressed per $10^{6}$ cells or per microliter of matrix volume. Thus mitochondria with higher GSH concentration exhibited greater UCR values, and the lower values of both UCR and GSH were found in PV hepatocytes. The relationships were similar when determining functional integrity as the ACR in relation to GSH (not shown). As seen in Fig. $6 \mathrm{~B}$, the results suggest that UCR declines when mitochondrial GSH fall below a threshold concentration.

\section{Discussion}

Mitochondrial GSH plays an important role in the maintenance of cellular functions and viability, as shown for a variety of cells and organs $(8,10,11)$. We have observed previously that ethanol induces a selective depletion of mitochondrial GSH, which results in an increased susceptibility to oxidant stress (8). The purpose of the present study was to address the relationship between mitochondrial GSH depletion and two important features of alcoholic liver disease, namely preferential PV injury and disturbances in mitochondrial function. Thus, to further support the significance of mitochondrial GSH depletion in pathogenesis of alcoholic liver disease, we hypothesize that changes in mitochondrial GSH would be greater in PV cells and would precede the appearance of disturbed mitochondrial function. To this purpose we have used zonally enriched PP and PV hepatocytes as well as isolated mitochondria from these zonally enriched cells.

We have observed an equal distribution of cytosol GSH between PP and PV hepatocytes which was unaffected by ethanol feeding. However, when the ethanol-induced enlargement of cells was taken into consideration, a moderate decrease in cytosol GSH concentration was estimated. This lower PV cytosol GSH concentration was not associated with a change in GSH synthetic capacity in intact or cell-free systems and thus probably reflects simple dilution of all constituents in GSH regulation, i.e., amino acids, synthetic enzymes, and feedback inhibition by GSH. Despite lower ATP levels in PV ethanol cells, ATP remains well above saturation for GSH synthetic enzymes (41). It is important to stress that this modest decrease in cytosol GSH cannot account for the degree of depletion of mitochondrial GSH (see Fig. 4). Basal levels of mitochondrial GSH from pair-fed cells show a modest trend for lower values in PV cells, which did not reach statistical significance. However, our data on the time-dependent accumulation of mitochondrial GSH and the relationship of mitochondrial to cytosol GSH showed similar results for PP and PV mitochondria from pairfed cells. In the pair-fed case, the only instance where there was a significant difference in mitochondrial GSH in PP versus PV cells was when content of GSH was expressed as absolute concentration, which probably reflects the slightly larger mito- 
Table IV. Cellular ATP Values and Mitochondrial Membrane Potential $\left(E_{m}\right)$ of PP and PV Cells from Pair-fed and Ethanol-fed Rats

\begin{tabular}{lrrrrr}
\hline & \multicolumn{2}{c}{ Pair-fed } & & \multicolumn{2}{c}{ Ethanol-fed } \\
\cline { 2 - 3 } \cline { 5 - 6 } & PP & PV & & PP & PV \\
\hline ATP $\left(n m o l / 10^{6}\right.$ cells $)$ & $20 \pm 3$ & $22 \pm 3.2$ & & $15 \pm 3$ & $11 \pm 4^{*}$ \\
$E_{\mathrm{m}}(m V)$ & $176 \pm 9$ & $181 \pm 12$ & & $158 \pm 11$ & $131 \pm 13^{*}$ \\
\hline
\end{tabular}

PP and PV hepatocytes were used to determine ATP levels by HPLC (37) and to prepare zonal-enriched mitochondria by Percoll centrifugation for mitochondrial membrane potential determination. Results are the mean \pm SD of $n=4$ for ATP and $n=3$ for $E_{\mathrm{m}}$ values. $* P<0.05$ vs pair-fed values by ANOVA.

chondria in PV versus PP cells. Even in this case it did not bear any impact on UCR values, suggesting that there seems to be a threshold phenomenon in which depletion of mitochondrial GSH below a certain level affects the mitochondrial functional integrity.

We have confirmed our earlier reports by showing a selective depletion of mitochondrial GSH in ethanol-fed hepatocytes. This defect is now shown to be more profound in PV cells on the basis of basal levels, rate and extent of repletion, and overall relationship among a wide range of cytosol GSH and mitochondrial GSH levels. Although chronic ethanol feeding also results in decreased mitochondrial GSH in PP cells, this is reflected in significant changes only in the mitochondrial functional integrity (UCR) without significant effects on ATP and membrane potential. This suggests that the sensitivity of mitochondrial parameters to GSH depletion is different for the UCR, ATP, and membrane potential. Therefore, the severe depletion of mitochondrial GSH in PV hepatocytes may contribute to the preferential injury to PV cells in alcoholic liver disease. Although cause and effect is very difficult to prove, we approached this issue by determining the relationship between mitochondrial GSH and mitochondrial function. We observed an uncoupling of oxidative phosphorylation in mitochondria from ethanol-fed

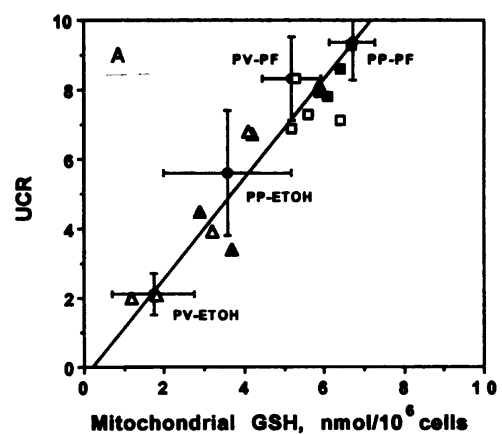

Figure 6. Correlation between GSH content and functional integrity of mitochondria. Mitochondria from PP and PV zones were prepared from pair-and ethanol-fed (PF and ETOH, respectively) rats by Percoll centrifugation as described in Methods. The mitochondrial GSH content was determined by the Tietze method (35) and was expressed as per $10^{6}$ cells $(A)$ or as millimolar using matrix volume $(B)$; the functional integrity of mitochondria was determined as the UCR as described in the legend to Fig. 5. Solid lines were determined by linear regression for results of $A$ and by polynomial equation for $B$. Results are the mean $\pm S D$ of $n=4$ preparations for each group. Resultsof Table $V$ were also included in the plot. Squares and triangles represent the mean of PP (solid) and PV (open) mitochondria prepared from animals fed pair (squares) or ethanol (triangles) liquid diet for 1-4 wk.

cells which was most pronounced in PV cells and correlated with decreased ATP and mitochondrial membrane potential. Indeed, the degree of impairment in functionality of isolated mitochondria correlated with mitochondrial GSH, being affected most severely in PV cells. Although we do not know the exact molecular basis for the close correlation between UCR and mitochondrial GSH, it has to be considered that $\mathrm{H}_{2} \mathrm{O}_{2}$, of which mitochondria are major producers, is hazardous to mitochondria because it can oxidize critical biomolecules directly or via formation of the highly reactive hydroxyl radical

Table V. Time Course of Effect of Chronic Ethanol Intake on GSH Content and Functional Integrity of PP and PV Mitochondria from Pair-fed and Ethanol-fed Cells

\begin{tabular}{|c|c|c|c|c|c|c|c|c|}
\hline \multirow[b]{3}{*}{ Wk } & \multicolumn{4}{|c|}{ Pair-fed } & \multicolumn{4}{|c|}{ Ethanol-fed } \\
\hline & \multicolumn{2}{|c|}{ PP } & \multicolumn{2}{|c|}{ PV } & \multicolumn{2}{|c|}{ PP } & \multicolumn{2}{|c|}{ PV } \\
\hline & GSH & UCR & GSH & UCR & GSH & UCR & GSH & UCR \\
\hline 1 & $6.4 \pm 0.6$ & $8.6 \pm 0.8$ & $5.6 \pm 0.8$ & $7.3 \pm 0.6$ & $\begin{array}{l}5.9 \pm 0.7 \\
(92 \pm 9)\end{array}$ & $\begin{array}{l}8.1 \pm 0.8 \\
(94 \pm 8)\end{array}$ & $\begin{array}{l}4.1 \pm 0.6^{*} \\
(73 \pm 7)\end{array}$ & $\begin{array}{l}6.8 \pm 0.5 \\
(92 \pm 9)\end{array}$ \\
\hline 2 & $5.9 \pm 0.8$ & $7.9 \pm 0.5$ & $5.2 \pm 1.1$ & $6.9 \pm 0.4$ & $\begin{array}{l}4.2 \pm 0.4 * \\
(71 \pm 7)\end{array}$ & $\begin{array}{l}6.7 \pm 0.6 \\
(78 \pm 9)\end{array}$ & $\begin{array}{l}3.2 \pm 0.4 * \\
(61 \pm 8)\end{array}$ & $\begin{array}{l}3.9 \pm 0.4^{*} \\
(57 \pm 7)\end{array}$ \\
\hline 3 & $6.1 \pm 0.9$ & $7.8 \pm 0.6$ & $6.4 \pm 0.7$ & $7.1 \pm 0.6$ & $\begin{array}{l}3.7 \pm 0.3^{*} \\
(60 \pm 5)\end{array}$ & $\begin{array}{l}3.4 \pm 0.4 * \\
(44 \pm 7)\end{array}$ & $\begin{array}{l}1.8 \pm 0.2 * \\
(28 \pm 5)\end{array}$ & $\begin{array}{l}2.1 \pm 0.2^{*} \\
(30 \pm 4)\end{array}$ \\
\hline 4 & $6.7 \pm 0.5$ & $9.3 \pm 0.6$ & $5.3 \pm 0.4$ & $8.3 \pm 0.6$ & $\begin{array}{l}2.9 \pm 0.2^{*} \\
(43 \pm 6)\end{array}$ & $\begin{array}{l}4.5 \pm 0.5 * \\
(64 \pm 7)\end{array}$ & $\begin{array}{l}1.2 \pm 0.4 * \\
(22 \pm 6)\end{array}$ & $\begin{array}{l}2.0 \pm 0.3 * \\
(24 \pm 5)\end{array}$ \\
\hline
\end{tabular}

Mitochondria from zonal-enriched cells were prepared from pair- and ethanol-fed rats for the period indicated to determine GSH content and UCR values. The numbers in parentheses indicate the percentage of control values. Results are the mean \pm SD of $n=4$ preparations. ${ }^{*} P<0.05$ vs pair-fed by ANOVA. 
(16). GSH, as a substrate for the glutathione peroxidase, detoxifies most mitochondrial $\mathrm{H}_{2} \mathrm{O}_{2}$ under normal conditions because of its abundance and low $K_{m}$ of GSH peroxidase for $\mathrm{H}_{2} \mathrm{O}_{2}$ and because, with the exception of heart, catalase is not found in mitochondria $(16,17)$. Therefore, elevated mitochondrial $\mathrm{H}_{2} \mathrm{O}_{2}$ due to mitochondrial GSH depletion could result in inactivation of respiratory chain carriers and other mitochondrial enzymes and in uncoupling of respiration $(42,43)$. Furthermore, examination of the influence of duration of ethanol exposure on these parameters indicates that the fall in mitochondrial GSH precedes the onset of changes in mitochondrial function, consistent with the fall in mitochondrial GSH contributing to the development of impaired oxidative phosphorylation. The molecular basis for impaired transport of GSH from cytosol to mitochondria as a consequence of ethanol exposure is at present unknown. The existence of inherent differences in the metabolism of ethanol within the liver acinus could account for the predominant impairment of the transport of GSH into the PV mitochondria. Acetaldehyde, the first by-product of the oxidative metabolism of ethanol, is a very toxic metabolite whose metabolism occurs exclusively in the mitochondria by the low $K_{\mathrm{m}}$ acetaldehyde dehydrogenase. We have found a lower enzymatic activity in control PV compared with PP cells, in agreement with a previously published work (30), which was decreased in both cells after ethanol feeding; thus, this would establish a gradient of acetaldehyde within the liver acinus that is more concentrated in the PV area. In this regard, it has been shown recently that ethanol in vitro inhibits the expression and transport of aldehyde dehydrogenase into liver mitochondria (44). The impact of the acetaldehyde gradient on the nuclear expression and import into mitochondrial membranes of the functional mitochondrial GSH carrier remains to be established. However, it is possible that acetaldehyde could affect the mitochondrial GSH levels not only by affecting the level of the expressed and imported mature carrier into mitochondria, but also by changing the physicochemical properties of mitochondrial membrane leading to altered function of the carrier. However, it should be noted that other mitochondrial transporters, i.e., ADP/Pi exchanger, ADP/ ATP translocator, NADPH shuttles, di and tricarboxylate exchangers, are altered minimally, if at all, by ethanol (45). It is possible that the fall in PV ATP levels may contribute to decreased GSH accumulation since Martensson et al. (46) have suggested that this is an active transport. However, this seems unlikely since PP cells showed moderately decreased mitochondrial GSH uptake without a fall in ATP levels. Thus, the impairment in the mitochondrial GSH transporter occurs early, is relatively specific, is preferentially seen in PV cells, and precedes alterations in mitochondrial function, which are also preferentially seen in PV cells. Thus, our results further support the likelihood that this is an important defect in contributing to the pathogenesis of alcoholic liver disease. The precise mechanism for the defect remains to be determined.

\section{Acknowledgments}

We highly appreciate Dr. Hipólito Fernández' assistance with the feeding of the rats.

This study was supported by grants from the National Institute of Alcohol Abuse and Alcoholism (AA09526 to N. Kaplowitz and J. C. Fernández-Checa), Dirección General Investigación Cientifica y Técnica (PB92-1110), and Europharma (to J. C. Fernández-Chera). Carmen García-Ruiz and Albert Morales were partially supported by a grant from Europharma.

\section{References}

1. Meister, A., and M. Anderson. 1983. Glutathione. Annu. Rev. Biochem. 52:711-760.

2. Kaplowitz, N., T. Y. Aw, and M. Ookhtens. 1985. The regulation of hepatic glutathione. Annu. Rev. Pharmacol. Toxicol. 25:715-744.

3. Fernández-Checa, J. C., S. Lu, M. Ookhtens, L. DeLeve, R. Kannan, M. Runnegar, H. Yoshida, H. Hideki, C. García-Ruiz, J. Kulenhkamp, and N. Kaplowitz. 1993. Regulation of hepatic GSH. In Hepatic Anion Transport and Bile Secretion: Physiology and Pathophysiology. N. Tavoloni and P. Berk, editors. Raven Press, New York. 345-395.

4. Sies, H., and A. Mosskim. 1978. Role of mitochondrial glutathione peroxidase in modulating mitochondrial oxidations in liver. Eur. J. Biochem. 84:377383.

5. Reed, D. J. 1986. Regulation of reductive processes by glutathione. Biochem. Pharmacol. 35:7-13.

6. Griffith, O. W., and A. Meister. 1985. Origin and turnover of mitochondrial glutathione. Proc. Natl. Acad. Sci. USA. 82:4668-4672.

7. Martensson, J., C. K. Lai, and A. Meister. 1990. High affinity transport of GSH is part of a multicomponent system essential for mitochondrial function. Proc. Natl. Acad. Sci. USA. 87:7185-7189.

8. Fernández-Checa, J. C., C. García-Ruiz, M. Ookhtens, and N. Kaplowitz. 1991. Impaired uptake of glutathione by hepatic mitochondria from chronic ethanol-fed rats. J. Clin. Invest. 87:397-405.

9. Kurosawa, K., N. Hayashi, N. Sato, T. Kamada, and N. Tagawa. 1991. Transport of GSH across mitochondrial membranes. Biochem. Biophys. Res. Commun. 167:367-372.

10. Martensson, J., and A. Meister. 1989. Mitochondrial damage in muscle occurs after marked depletion of GSH and is prevented by giving GSH-ester. Proc. Natl. Acad. Sci. USA. 86:471-475.

11. Martensson, J., A. Jain, W. Frayer, and A. Meister. 1989. Glutathione metabolism in the lung: inhibition of its synthesis leads to lamellar body and mitochondrial defects. Proc. Natl. Acad. Sci. USA. 86:5296-5300.

12. Meredith, M., and D. J. Reed. 1988. Depletion in vitro of mitochondrial GSH in rat hepatocytes and enhancement of lipid peroxidation by adriamycin and BCNU. Biochem. Pharmacol. 32:1383-1388.

13. Lotscher, H. R., K. Winterhalter, E. Carafoli, and C. Richter. 1980. Hydroperoxide-induced loss of pyridine nucleotide and release of calcium from rat liver mitochondria. J. Biol. Chem. 255:9325-9330.

14. Neubert, D., A. B. Wojtszak, and A. L. Lehninger. 1962. Purification and enzymatic identity of mitochondrial contraction factors I and II. Proc. Natl. Acad. Sci. USA. 48:1651-1658.

15. Lehninger, A. L., A. Vercesi, and E. Bababumni. 1980. Regulation of $\mathrm{Ca}^{2+}$ release from mitochondria by the oxidation-reduction state of pyridine nucleotides. Proc. Natl. Acad. Sci. USA. 75:1690-1694.

16. Chance, B., H. Sies, and A. Boveris. 1979. Hydroperoxide metabolism in mammalian organs. Physiol. Rev. 59:527-605.

17. Radi, R., J. F. Turrens, L. Y. Chang, K. M. Bush, J. D. Crapo, and B. A. Freeman. 1991. Detection of catalase in rat heart mitochondria. J. Biol. Chem. 266:22028-22034.

18. Fernández-Checa, J. C., M. Ookhtens, and N. Kaplowitz. 1987. Effect of chronic ethanol feeding on rat hepatocytic glutathione. Compartmentation, efflux, and response to incubation with ethanol. J. Clin. Invest. 80:57-62.

19. Fernández-Checa, J. C., M. Ookhtens, and N. Kaplowitz. 1989. Effects of chronic ethanol feeding on rat hepatocytic glutathione. Relationship of cytosolic glutathione to efflux and mitochondrial sequestration. J. Clin. Invest. 83:12471252.

20. Takeshi, H., N. Kaplowitz, T. Kamimura, H. Tsukamoto, and J. C. Fernández-Checa. 1992. Hepatic mitochondrial GSH depletion and progression of experimental alcoholic liver disease in rats. Hepatology. 16:1423-1428.

21. Kiessling, K. H., and U. Tobe. 1964. Degeneration of liver mitochondria in rats after prolonged alcohol consumption. Exp. Cell Res. 33:350-354.

22. Porta, E. A., W. S. Hartroft, and F. A. de la Iglesia. 1965. Hepatic changes associated with chronic alcoholism in rats. Lab. Invest. 14:1437-1455.

23. Bruguera, M., A. Bertán, J. A. Bombi, and J. Rodés. 1977. Giant mitochondria in hepatocytes. A diagnostic hint for alcoholic liver disease. Gastroenterology. 73:383-387.

24. Cederbaum, A. I., and E. Rubin. 1975. Molecular injury to mitochondria produced by ethanol and acetaldehyde. Fed. Proc. 34:2045-2051.

25. Thayer, W. S., and E. Rubin. 1979. Effects of chronic ethanol intoxication on oxidative phosphorylation in rat liver submitochondrial particles. J. Biol. Chem. 254:7717-7723.

26. Popper, H., and C. S. Lieber. 1980. Histogenesis of alcoholic fibrosis and cirrhosis in the baboon. Am. J. Pathol. 98:695-716.

27. DeCarli, L. M., and C. S. Lieber. 1967. Fatty liver in the rat after prolonged intake of ethanol on nutritionally adequate new liquid diet. J. Nutr. 91:131-138.

28. Lindros, K. O., and K. E. Penttila. 1985. Digitonin-collagenase perfusion for efficient separation of periportal and perivenous hepatocytes. Biochem. $J$. 228:757-760. 
29. Moldeus, P., P. Hodberg, and S. Orrenius. 1978. Isolation and use of liver cells. Methods Enzymol. 51:60-70.

30. Cheng, L., R. R. Sidner, and L. Lumeng. 1992. Distribution of alcohol dehydrogenase and the low $K_{\mathrm{m}}$ form of aldehyde dehydrogenase in isolated perivenous and periportal hepatocytes in rats. Alcohol. Clin. Exp. Res. 16:23-29.

31. Braakman, I., J. Keij, M. J. Hardonk, D. K. F. Meijer, and G. M. M. Grothius. 1991. Separation of periportal and perivenous rat hepatocytes by fluorescence-activated cell sorting: confirmation with colloidal gold as an exogenous marker. Hepatology. 13:73-82.

32. Reinhart, P. H., W. M. Taylor, and F. L. Bygrave. 1982. A procedure for the rapid preparation of mitochondria from rat liver. Biochem. J. 204:731-735.

33. Fernández-Checa, J. C., and N. Kaplowitz. 1990. The use of monochlorobimane to determine hepatic GSH levels and synthesis. Anal. Biochem. 190:212219.

34. Porter, R. K., J. M. Scott, and M. D. Brand. 1992. Choline transport into rat liver mitochondria. Characterization and kinetics of a specific transporter. $J$. Biol. Chem. 267:14637-14641.

35. Tietze, F. 1969. Enzymatic method for quantitative determination of nanogram amounts of total and oxidized glutathione: application to mammalian blood and other tissues. Anal. Biochem. 77:502-522.

36. Fariss, M. W., and D. J. Reed. 1987. High performance liquid chromatography of thiols and disulfides: dinitrophenol derivatives. Methods Enzymol. 143:101-109.

37. Jones, D. P. 1981. Determination of pyridine nucleotides in cell extracts by high-performance liquid chromatography. J. Chromatogr. 225:446-449.
38. Hatefi, Y., and D. L. Stigall. 1978. Preparation and properties of succinate: ubiquinone oxidoreductase (complex II). Methods Enzymol. 53:21-27.

39. Wellner, V. P., and A. Meister. 1966. Binding of adenosine triphosphate and adenosine diphosphate by glutamine synthetase. Biochemistry. 5:872-879.

40. Fernández-Checa, J. C., C. Ren, T. Y. Aw, M. Ookhtens, and N. Kaplowitz. 1988. Effect of membrane potential and cellular ATP on glutathione efflux from isolated hepatocytes. Am. J. Physiol. 255:G403-G408.

41. Shan, T., T. Y. Aw, R. Shapiro, and D. P. Jones. 1989. Oxygen-dependence of glutathione synthesis in hepatocytes. Toxicol. Appl. Pharmacol. 101:261 -270.

42. Radi, R., K. M. Bush, and B. A. Freeman. 1993. The role of cytochrome $c$ and mitochondrial catalase in hydroperoxide-induced heart mitochondrial lipid peroxidation. Arch. Biochem. Biophys. 300:409-415.

43. Zhang, Y., O. Marcillat, C. Giulivi, L. Ernster, and K. L. Davies. 1990. The oxidative inactivation of mitochondrial electron transport chain components and ATPase. J. Biol. Chem. 265:16330-16336.

44. Wang, T. Y., J. Farres, and H. Weiner. 1989. Liver mitochondrial aldehyde dehydrogenase: in vitro expression, in vitro import and effect of alcohols on import. Arch. Biochem. Biophys. 272:440-449.

45. Cunningham, C. C., W. B. Coleman, and P. I. Spach. 1990. The effects of chronic ethanol consumption on hepatic mitochondrial energy metabolism. Alcohol Alcohol. 25:127-136.

46. Martensson, J., J. C. K. Lai, and A. Meister. 1990. High-affinity transport of glutathione is part of a multicomponent system essential for mitochondrial function. Proc. Natl. Acad. Sci. USA. 87:7185-7189. 